\title{
Big Data and Situation-Aware Technology for Smarter Healthcare
}

\author{
Mu-Yen Chen ${ }^{1} \cdot$ Edwin David Lughofer ${ }^{2} \cdot$ Robi Polikar $^{3}$
}

Published online: 30 October 2018

(C) Taiwanese Society of Biomedical Engineering 2018

\section{Introduction}

This special issue investigates the application of big data analysis and situation-aware technology in healthcare and related innovations. Interaction between technology and healthcare has emerged as a critical area of research, with the rapid development of innovations including Internet of Things (IoT), big data analysis techniques, and miniature wearable biosensors generating new opportunities for situation-aware mHealth and uHealth healthcare systems. Wearable health sensors provide many advantages for location-aware, situation-aware, mobile and ubiquitous data collection and real-time processing of big data. In addition, methods for raw data collection, data preprocessing, data storage, and data mining have become increasingly efficient for very large data sets.

However, many challenges still remain to be addressed in the development of consistent, suitable, safe, flexible and real-time healthcare systems. These issues include the streaming nature of data collection in IoT environments and situation-aware care deficiencies. The current issue explores how individual caregivers or entire hospitals can benefit from using novel situation-aware technologies in real-world settings through the application of big data analysis techniques. It also encourages further research, using empirical studies to present application of fundamental theory, new

Mu-Yen Chen

mychen@nutc.edu.tw

Edwin David Lughofer

Edwin.Lughofer@jku.at

Robi Polikar

polikar@rowan.edu

1 Department of Information Management, National Taichung University of Science and Technology, Taichung, Taiwan

2 Department of Knowledge-Based Mathematical Systems, Johannes Kepler University Linz, Linz, Austria

3 Department of Electrical and Computer Engineering, Rowan University, Glassboro, NJ, USA techniques, and practical experience in the context of smart healthcare-wearable sensors, body area sensors, IoT, and big data analytics that take advantage of advances in the bioinformatics domain.

\section{Fewer Research Questions, Diverse Fields}

The first field covers Big Data Analysis. Liu et al. [1] propose a novel ECG signal enhancement approach for big data based on significant similarities calculations. Experimental results show the proposed method outperformed wavelet with subband dependent thresholding (WT-Subband), Back Propagation Neural Networks (BPNN) and the Stockwell transform. Chan et al. [2] construct a tissue section imagebased liver scar stage (TSILSS) diagnosis system to investigate stages of liver scarring. Experimental results show an average prediction accuracy as high as $90 \%$, providing physicians with a valuable diagnositic tool. Ural [3] presents a brain tumor detection system based on magnetic resonance imaging (MRI) which can also detect and identify tumor areas in the brain using probabilistic neural networks (PNN). Experimental diagnostic results show this approach obtains high classification accuracy from image processing and appropriate neural network structures in big data environments. Manohar and Ganesan [4] investigate the specific texture features of schizophrenic MR images, with experimental results showing better accuracy than traditional classification methods. Lin et al. [5] develop a novel EEG classification algorithm to identify normal, spike, and seizure EEG signals with high accuracy. Abdar et al. [6] construct an early detection system integrating Multilayer Perceptron Neural Network (MLPNN) with various classification algorithms. The proposed hybrid model is found to effectively predict and classify liver disease. Al Rahhal et al. [7] applied convolutional neural networks (CNN) to handle very large labeled images using a deep learning approach to accurately detect ventricular ectopic beats (VEB) and supraventricular ectopic beats (SVEB). Lu et al. [8] propose a modification confidence measure algorithm to improve the accuracy of 
bio-image denoising processes, showing significant performance improvements especially in sliding window processes from the top-left to the bottom-right of bio-images. Finally, Guzmán et al. [9] present a collaborative framework for identifying physiological variables to identify high risk cardiovascular conditions. Experimental results show the proposed model can achieve higher accuracy than the popular ID3, J48, NBTree, and BFTree algorithms.

The second field focuses on Situation-aware and Intelligent Systems. Chiang et al. [10] apply singular value decomposition (SVD), Fourier transform (FT), minimum entropy principle, and associative Petri net to analyze EEG signals and build an attention evaluation system. Aktas et al. [11] construct a novel hospital information system based on IoT approaches with WBANs and RFID. The resulting system provides a decision-making reference to support IoT-based health monitoring and management. Ranjan et al. [12] report an eye blink tracking system that can not only receive EEG signal sequences smoothly but also successfully extract important eye blinks. Chiang and Chen [13] propose a home care system for elderly suffering from the Three-High Diseases, and also develop a mobile App-based service to automatically send alert messages to family members and medical staff. Awojoyogbe and Dada [14] propose a novel approach using magnetic resonance relaxation for bioheat transfer. Huang et al. [15] apply radiofrequency ablation (RFA) to fluid saline injection to expand the coagulation area and demonstrate the influence of both electrical conductivity and blood perfusion rate with different parameter settings.

\section{Conclusion}

The application of information technologies to the medical domain raises opportunities for the development of new diagnostics and treatments, making it a critical area of investigation. The editors gratefully acknowledge the many high quality submissions received, and the generous effort of all reviewers. We hope this special issue serves to spark new discussion, exploration and collaboration.

\section{References}

1. Liu, M., Hao, H., Xiong, P., et al. (2018). Constructing a guided filter by exploiting the butterworth filter for ECG signal enhancement. Journal of Medical and Biological Engineering. https://doi. org/10.1007/s40846-017-0350-1.
2. Chan, Y. K., Chang, M. J., Hung, Y. W., et al. (2017). Tissue section image-based liver scar detection. Journal of Medical and Biological Engineering. https://doi.org/10.1007/s40846-017-0352-z.

3. Ural, B. (2017). A computer-based brain tumor detection approach with advanced image processing and probabilistic neural network methods. Journal of Medical and Biological Engineering. https:// doi.org/10.1007/s40846-017-0353-y.

4. Manohar, L., \& Ganesan, K. (2017). Diagnosis of schizophrenia disorder in MR brain images using multi-objective BPSO based feature selection with Fuzzy SVM. Journal of Medical and Biological Engineering. https://doi.org/10.1007/s40846-017-0355-9.

5. Lin, J. W., Chen, W., Shen, C. P., et al. (2018). Visualization and sonification of long-term epilepsy electroencephalogram monitoring. Journal of Medical and Biological Engineering. https://doi. org/10.1007/s40846-017-0358-6.

6. Abdar, M., Yen, N. Y., \& Hung, J. C. S. (2018). Improving the diagnosis of liver disease using multilayer perceptron neural network and boosted decision trees. Journal of Medical and Biological Engineering. https://doi.org/10.1007/s40846-017-0360-z.

7. Al Rahhal, M. M., Bazi, Y., Al Zuair, M., et al. (2018). Convolutional neural networks for electrocardiogram classification. Journal of Medical and Biological Engineering. https://doi. org/10.1007/s40846-018-0389-7.

8. Lu, C. T., Shen, J. H., Wang, L. L., et al. (2017). Impulse noise denoising using confidence measure with non-sequential process order for X-ray bio-images. Journal of Medical and Biological Engineering. https://doi.org/10.1007/s40846-017-0356-8.

9. Guzmán, G., Torres-Ruiz, M., Tambonero, V., et al. (2018). A collaborative framework for sensing abnormal heart rate based on a recommender system: Semantic recommender system for healthcare. Journal of Medical and Biological Engineering. https ://doi.org/10.1007/s40846-018-0421-y.

10. Chiang, H. S., Hsiao, K. L., \& Liu, L. C. (2017). EEG-based detection model for evaluating and improving learning attention. Journal of Medical and Biological Engineering. https://doi. org/10.1007/s40846-017-0344-z.

11. Aktas, F., Ceken, C., \& Erdemli, Y. E. (2018). IoT-based healthcare framework for biomedical applications. Journal of Medical and Biological Engineering. https://doi.org/10.1007/s4084 6-017-0349-7.

12. Ranjan, R., Arya, R., Kshirsagar, P., et al. (2018). Real time eye blink extraction circuit design from EEG signal for ALS patients. Journal of Medical and Biological Engineering. https://doi. org/10.1007/s40846-017-0357-7.

13. Chiang, T. C., \& Chen, C. H. (2017). Intelligent three-high diseases home warning system based on fuzzy theory. Journal of Medical and Biological Engineering. https://doi.org/10.1007/ s40846-017-0368-4.

14. Awojoyogbe, B. O., \& Dada, M. O. (2018). Computational design of an RF controlled theranostic model for evaluation of tissue biothermal response. Journal of Medical and Biological Engineering. https://doi.org/10.1007/s40846-018-0386-x.

15. Huang, H. W., Hui, L., Hung, J. C., et al. (2017). Illustrating the impact of uneven saline distribution on thermal lesion during radiofrequency ablation using computer simulation for smarter healthcare treatment planning. Journal of Medical and Biological Engineering. https://doi.org/10.1007/s40846-017-0354-x. 